\title{
Pharmacists in a Complex Chronic Disease Management Clinic
}

\author{
Uzma Alim, Nyanza Austin-Bishop, and Ginny Cummings
}

\section{INTRODUCTION}

I

magine the following case scenario: Mrs $\mathrm{K}$ is a 67-year-old 1 woman with congestive heart failure, chronic kidney disease, poorly controlled type 2 diabetes mellitus, gastroesophageal reflux disease, and atrial fibrillation. She has had 3 admissions to hospital over the past 6 months, related to both acute kidney injury and congestive heart failure. The most recent admission was complicated by hospital-acquired pneumonia requiring broad-spectrum antibiotics and by Clostridium difficile infection, which resulted in a 4-week hospital stay and significant deconditioning. Now, she is finally ready for discharge, and the internal medicine team is arranging required follow-up with her family physician, the heart function clinic, an endocrinologist, a nephrologist, and the anticoagulation management service. Because of her frequent hospital admissions, Mrs K has missed several standing appointments with specialists and has not yet been connected with the home care services that she will need after discharge.

Does this scenario sound familiar? Like many patients with complicated medical needs, Mrs $\mathrm{K}$ is overwhelmed by her conditions, her medications, a myriad of appointments, and questions about what she can do to improve her health and stay out of hospital. It is for patients like this, who are high users of the health care system, that a new clinic was created at the Peter Lougheed Centre in Calgary, Alberta.

The Complex Chronic Disease Management Clinic (CCDMC) is a multidisciplinary ambulatory clinic staffed by nurse clinicians, pharmacists, and internal medicine physicians. It was established in 2008 to enhance access to specialized medical services for patients with a history of multiple hospital admissions and complex care needs. Its purpose is to reduce exacerbations of chronic conditions, visits to the emergency department, and acute care admissions, through intensive disease management. Preliminary data from the first year of operation showed that the clinic decreased by one-quarter the number of hospital admissions and reduced by half the length of stay for those who did need admission. ${ }^{1}$ Updated data are currently being collected to demonstrate the ongoing effectiveness of the clinic. The current article describes the structure of the clinic, as well as the role of the pharmacist within the clinic team.

The CCDMC was modelled on the Alfred model of chronic complex disease management, ${ }^{2}$ which postulates that providing services through one ambulatory clinic is a better use of resources and yields better patient satisfaction than seeing patients in multiple speciality clinics. All of the patients at the CCDMC have multiple comorbid conditions, often struggle with complications such as disability or psychosocial issues, and are frequent users of acute care services. Patients are eligible for this clinic if they have 2 or more chronic disease conditions (e.g., asthma, hypertension, congestive heart failure, dyslipidemia, diabetes), excluding chronic pain and kidney disease requiring dialysis, and if they have had at least one hospital admission or emergency department visit in the past year. Referrals come primarily from physicians at urban Calgary hospitals who would like to have their patients with complex needs closely followed after discharge. Referrals may also be made by family physicians, home care nurses, or other ambulatory clinics. Patients are typically local to Calgary or the surrounding geographic area.

\section{CLINIC TEAM}

The multidisciplinary team at the CCDMC sees patients 3 mornings per week, and the nurse clinicians are available by telephone on all weekdays. In addition, community paramedics have recently been integrated into the program to allow for in-home assessments on both weekdays and weekends for patients who may be medically fragile. According to 20122016 data, the CCDMC receives roughly 100 new referrals each year, and staff members see 15-20 patients per week. The 
waiting time between referral and a patient's first appointment is typically between 1 and 3 weeks, and as of late 2016, the CCDMC was handling about 140 active cases. A maximum of 6 patients are booked for appointments at the clinic each day (with the typical number of patients per day ranging between 4 and 6), and each patient visit lasts 1 to 2 hours. The frequency of visits per patient depends on the patient's needs and varies from every 2 weeks to every 3 months.

On each clinic day, the CCDMC clinic team consists of 2 nurse clinicians, 1 internal medicine physician (from a group of 12 internal medicine physicians), and 1 pharmacist. The clinic process involves one of the nurse clinicians and the pharmacist first assessing a new patient together, with the nurse clinician focused on performing a physical assessment and the pharmacist focused on compiling a comprehensive medication history. The nurse clinician and the pharmacist then discuss key findings and issues with the physician. On the basis of their discussion, this team decides on a plan and then meets with the patient to discuss the plan and specific recommendations. If there are any new medication orders or medication-related changes, the pharmacist is responsible for entering prescriptions and updating the patient's outpatient medication list using an electronic medical record and documentation system. Because of time constraints, the pharmacist triages which patients to see at subsequent visits, working with the nurse clinicians to prioritize those with unresolved medication management issues and those who require pharmacist review of their medications. Patients with previously identified medication issues requiring follow-up and those with recent medication changes or hospitalization would have high priority to be seen by the pharmacist.

Communication between the CCDMC and the patient's family physician is crucial to long-term management of the patient. Each patient is expected to receive ongoing care from the family physician concurrently with clinic care, and the CCDMC also helps to ensure that recommendations from all of the patient's health care providers are implemented. Patients may selectively continue to see specialist physicians, but the CCDMC critically reviews and discusses with the patient which appointments might be discontinued without adverse consequence. We have found that most specialist follow-up appointments can be replaced by monitoring in the CCDMC, unless ongoing diagnostic tests are being arranged or completed. Disagreements with the advice of other providers and contradictory treatment plans are discussed thoroughly with patients to determine the most appropriate course of action, with clear communication and rationale being documented and shared broadly. After each patient visit, all care plans and recommendations are documented, and the clinic notes are shared with the patient's other care providers. As well, the patient receives an instruction sheet listing all recommendations and medication changes in patient-friendly language.

\section{ROLE OF THE PHARMACIST}

Studies have shown that integrating pharmacists into ambulatory clinics can improve chronic disease management and optimal use of medications. ${ }^{3}$ Pharmacists at the CCDMC play a vital role as medication therapy management experts. Currently, a 0.6 full-time equivalent pharmacist position is assigned to the clinic, and 3 pharmacists (including N.A.B. and G.C.) rotate through the clinic every 2 to 3 weeks, in addition to their roles in other clinics and departments. The pharmacists report to an ambulatory pharmacy manager in the Calgary zone of Alberta Health Services and receive professional development support from a clinical practice leader. The pharmacists use electronic documentation in the medical record, as well as a paper summary monitoring sheet to track each patient's medication history and compliance with various key evidencebased therapies. The CCDMC pharmacists are responsible for a wide variety of patient-centred activities, including medication reconciliation; detecting drug interactions; ordering laboratory tests to monitor drug therapy; prescribing, adapting, and extending prescriptions; and providing patient education. Seamless care to communicate medication-related changes to community pharmacists, as well as to hospital pharmacists when CCDMC patients are admitted, is another important role. Pharmacists at the CCDMC also participate in teaching pharmacy students from the University of Alberta and serve as preceptors for the ambulatory rotation requirement of the Alberta Health Services pharmacy practice residency program.

In addition, all of the pharmacists at the CCDMC have qualified for additional prescribing authorization and use this qualification to manage new and ongoing drug therapy. More specifically, since 2007, pharmacists in Alberta with additional prescribing authorization have been allowed to prescribe prescription-only drugs (excluding narcotics and controlled substances) on the basis of their initial assessment of a patient or in collaboration with either another authorized prescriber or a regulated health professional with nonprescriptive authority. ${ }^{4}$ According to a recent qualitative study of the prescribing practices of Alberta pharmacists, primary care pharmacists participate mainly in disease-focused prescribing (where current therapies are adapted or initiated according to a protocol in a specific therapeutic area) or patient-focused prescribing (where therapy is initiated on the basis of the patient's needs and values, a patient assessment, and best evidence). ${ }^{5}$ At the CCDMC, pharmacists mainly practise patient-focused prescribing; there are no specific prescribing protocols in place. All prescribing decisions are collaborative and tailored to each patient's individual needs. Medications commonly prescribed by the CCDMC pharmacists include diabetes medications, antihypertensives, iron supplements, anticoagulants, and gastrointestinal agents. As well, the pharmacists are strong advocates for thoughtful de-prescribing of medications that may no longer be indicated or may no longer be of benefit to the patient. 
Over the past few years, the role of the pharmacist at the CCDMC has evolved to also include assisting patients as they navigate the health care system, connecting them with community-based health care resources and encouraging them to practise self-care (e.g., motivating patients to have a greater understanding of their medications, encouraging patients with diabetes to self-monitor their blood glucose levels). Two of the 3 pharmacists have been trained in physical assessment skills, and the clinic is exploring opportunities for pharmacists to obtain vital signs and perform a basic physical exam during patient visits. Although pharmacists do not have any time or funding allocated for patient follow-up outside of clinic hours, they often work on their nonclinic days to follow up by telephone about patient-specific medication issues arising from recent visits or upon request from the nurse clinicians. Patientrelated workload metrics for time spent both in clinic and outside of clinic time are recorded, and it is hoped that these data can eventually be used to secure funding for additional staffing. In addition, the CCDMC team is currently conducting research to further describe the complexity of the cases seen at this clinic and to determine the clinic's impact in helping patients to achieve evidence-based outcomes for common chronic disease states.

\section{CONCLUSION}

So what could the CCDMC offer Mrs K, the patient described at the beginning of this article? The nurse clinician in the clinic can work closely with this patient to ensure that home care is arranged and can communicate with her other health care providers to perhaps reduce the need for further specialty clinic appointments. Referral to selected and easily accessible community-based nutrition, diabetes, and active living or rehabilitation programs can be initiated within the CCDMC, with follow-up to ensure that these are successful and are meeting Mrs K's needs. The pharmacist can complete a comprehensive medication review and can perform ongoing monitoring and adjustment of her medications, with an eye to discontinuation of nonessential medications, in accordance with Mrs K's wishes. Between clinic visits, Mrs K may call the nurse clinician with any concerns.
We believe that the interprofessional model of the CCDMC, as well as the clinic's focus on patients with multiple complex medical conditions, distinguishes it from typical ambulatory clinics within Canada. The clinic model supports the vision for Alberta Health Services pharmacists to provide high-quality pharmacy care through a team dedicated to collaboration, professionalism, and continual learning. The role of CCDMC pharmacists exemplifies how pharmacists can make a meaningful contribution to patient care and pursue their maximum scope of clinical practice.

References

1. The most complicated cases: managing complex chronic diseases. Ottawa $(\mathrm{ON})$ : Canadian Nurses Association; 2012 [cited 2016 May 6]. Available from: https://www.cna-aiic.ca/ -/media/cna/page-content/pdf-en/chronic_care_e. pdf?la=en

2. Linden A, Bonollo M, Fiddes K. A successful hospital-based disease management program to reduce admissions among patients with multiple chronic illnesses. Int J Pers Cent Med. 2011;1(4):675-82.

3. Tan EC, Stewart K, Elliott RA, George J. Pharmacist services provided in general practice clinics: a systematic review and meta-analysis. Res Social Adm Pharm. 2014;10(4):608-22.

4. Standards of practice for pharmacists and pharmacy technicians. Edmonton (AB): Alberta College of Pharmacists; 2011 [cited 2016 May 6]. Available from: https://pharmacists.ab.ca/sites/default/files/StandardsOfPractice.pdf

5. Guirguis LM, Makowsky MJ, Hughes CA, Sadowski CA, Schindel TJ, Yuksel N. How have pharmacists in different practice settings integrated prescribing privileges into practice in Alberta? A qualitative exploration.J Clin Pharm Ther. 2014;39(4):390-8.

Uzma Alim, PharmD, ACPR, was, at the time of writing, a Pharmacy Resident with Alberta Health Services, Calgary, Alberta. She is now a Pharmacist with St Joseph's Health Centre, Toronto, Ontario.

Nyanza Austin-Bishop, BScPharm, ACPR, CGP, is a Clinical Pharmacist with Alberta Health Services, Calgary, Alberta.

Ginny Cummings, BSCPharm, ACPR, is a Pharmacy Clinical Practice Leader, Alberta Health Services, Calgary, Alberta.

Competing interests: None declared.

Address correspondence to:

Ginny Cummings

Pharmacy Services, Alberta Health Services

Room 3689, Peter Lougheed Center

350026 Avenue NE

Calgary AB T1Y 6J4

e-mail: ginny.cummings@ahs.ca

Funding: None received. 